OPEN ACCESS

Edited by:

Steve Suib,

The University of Connecticut,

United States

Reviewed by:

Bryce Sadtler

Washington University in St. Louis,

United States

Benjamin T. Diroll,

Argonne National Laboratory (DOE),

United States

*Correspondence:

Fan Zhang

zhang_fan@fudan.edu.cn

Specialty section:

This article was submitted to

Nanoscience,

a section of the journal

Frontiers in Chemistry

Received: 03 August 2017 Accepted: 28 September 2017

Published: 31 October 2017

Citation:

Zhang F (2017) Grand Challenges for Nanoscience and Nanotechnology in

Energy and Health.

Front. Chem. 5:80.

doi: 10.3389/fchem.2017.00080

\section{Grand Challenges for Nanoscience and Nanotechnology in Energy and Health}

\author{
Fan Zhang * \\ Department of Chemistry, Fudan University, Shanghai, China
}

Keywords: nanotechnology, solar energy, nanomaterials, nanostructures, hydrogen energy, biomedicine, energy conversion, energy storage

During the past decade, new directions of modern research, broadly defined as nanoscale science and technology have emerged. Nanotechnology is not a separate scientific field, it is a complex platform for the existing disciplines of chemistry, physics, biology, medicine, neurology, information technology, and engineering, a new multidisciplinary scientific research area. In recent years, the nanotechnology has attract a great deal of attention in both synthesis methodologies and wide applications of medicine, energy, environmental, electronics etc. Despite of significant progress in nanotechnology and rise of many commercialized products involving nanomaterials, nanoscience, and technology are still facing many new challenges, especially in the areas of great concern to the public: energy and health.

\section{NANOMATERIALS FOR ENERGY CONVERSION}

As versatile components of optoelectronic devices, the nanostructured materials attracted a great deal of attention due to its unique ability to manipulate light and control energy flow at nearly the atomic level (Ge and Yin, 2011; Linic et al., 2011). Actually, most of the so called next generation solar cells are based on the nanostructured materials, include those that are based on nanomaterials and/or nanostructures such as quantum dot solar cells, nanowire, mesoscopic nanostructures, and so on (Beard et al., 2012, 2014). They exhibit great promise of new conception or routes for converting solar energy into some other types of energy, such as electronic (Masuko et al., 2014; Mei et al., 2014), chemical fuels (Reece et al., 2011; Thomann et al., 2011), etc. However, there are still many challenges need to be addressed before they were fully used in the practical applications.

Most of today's commercial solar cells with typically efficiency of $10-25 \%$ are based the silicon (Green et al., 2015). To make solar energy economically competitive with other energy sources, it is critical find ways to lower the solar cell's costs by improve the efficiency. The nanotechnology provides us a valuable clue, because the nanomaterials can exhibit quite different and new properties compared with the corresponding bulk materials, which allow us to develop new ways to convert the solar energy into electricity or fuels (Nozik et al., 2010). Generally, there are three broad routes based on nanostructures are used to decrease the cost of solar cells (Shockley and Queisser, 1961): (1) decrease the usage of the materials, for example fabrication of the thin film solar cells; (2) increase the efficiency of the photovoltaic devices, for example fabrication of the multi-junction solar cells; (3) increase the stability and life cycle of the device. All the methods, individually or in combination, can lead greatly lower the costs of the solar energy.

For the front surface reflectivity in solar cell devices, nanostructured surfaces can effectively enhance the anti-reflection ability when the diameter of the nanostructure decreased below the wavelength of the incident light. If the active components can be patterned to the anti-reflection nanostructure, the usage of the solar light can be great enhanced, and the costly 
anti-reflection coatings and texturing can be avoided (Oh et al., 2012). In addition, because the patterned nanostructures can capture more light via light trapping so that less material is needed to absorb the solar flux (Garnett and Yang, 2010; Kelzenberg et al., 2010). New photovoltaic nanotechnologies should not only possess the potential to reduce the cost of module, but also to achieve power conversion efficiency beyond 33\% (Shockley-Queisser Efficiency Limit; Shockley and Queisser, 1961). Both the more efficient usage of high-energy photons and using the normally not converted photons with low energy (upconversion) can achieve higher limiting efficiencies. The two approaches to eliminate the energy losses are related the exploring of novel nanostructures (Beard et al., 2009, 2012; Choi et al., 2011; Wang et al., 2011; Krogstrup et al., 2013; Cirloganu et al., 2014). The device stability is another important factor need to be considered in the photovoltaic technologies for the decreasing of the cost of the solar energy (Zhang et al., 2013; Chuang et al., 2014; Ning et al., 2014).

The nanotechnology is a double-edged sword, some of the features that benefit to solar cells may also introduce additional challenges at the same time. The influence of surfaces and interfaces on recombination pathways are more important for nanocrystals due to its large surface/volume ratio. The migration of photo-excited charges can be hindered across nanocrystal grains, and the increased amount of surface states can deactivate the charge carriers. So, in a nanocrystal solid device, where each individual nanocrystal carries size dependent properties of the nanomaterials, the transport of charges is dominated by the interparticle medium. The surface ligands of the colloidal nanostructures should be well designed: (i) to make sure the stabilization of the colloidal stabilization, (ii) provide facile and stable charge transportation between the nanocrystals, and (iii) constructively supplement the properties of the nanocrystal solid.

While the new generation of photovoltaic cells (thinfilm chalcogenides, hybrid perovskite materials, multi-junction solar cells, quantum dot-based solar cells, polymer solar cells etc.) exhibit great promise in the applications, it also presents challenges in the practical application. The challenges can be addressed in the following directions: (1) a simple fabrication processing with low manufacturing complexities and costs. Solution-based deposition and processing by using the nanoparticles inks is an appealing route. It has attracted a great deal of attention in the energy conversion, due to its unique properties including atmospheric pressure and low temperature processing, compatibility for the flexible substrates and largearea, high throughput, and so on. In addition, these approaches can be readily adapted for patterning materials without any subsequent processing steps. (2) One-dimensional nanomaterials can also offered new opportunities to design more efficient solar cells for enhancing solar cell efficiencies. By facilitating electron collection, transport and photon absorption, the onedimensional nanostructures, including nanorods, nanotubes, nanowires etc. provide significant opportunities for improving solar cell efficiency. (3) The development of new materials with not only high photon absorption, electron transport, etc. but also heavy metal free is much more important for the practical application, because the many of the present energy conversion nanomaterial, such as perovskites, CdTe share a common disadvantage of environmentally hazardous heavy metal, which hinder the commercialization of them.

Beside the energy conversion, the energy economical consumption is also very important for the energy sustainable development. Among the straightforward successes of nanotechnology familiar to consumers, the light emitting devices (LED) with high electron to light conversion efficiency have been well developed in the past decade. The high luminescence efficiency and uniquely size-tunable color of solution processable semiconducting colloidal quantum dots (QDs) highlight the potential of QDLEDs for use in energyefficient, solid state lighting and high color quality thin-film display. The key advantages of using quantum dots QDs in display and lighting applications, including their color purity, stability, and solution processability. From a device efficiency perspective, the three key challenges facing by QDLEDs are QDs photoluminescence quenching, poor photon outcoupling, and a limited understanding of the fundamental operating mechanisms of QDLEDs. Additionally, if the QDLED are to become a broad commercial reality, the operational lifetime and cost of QDLEDs must be addressed in the further research.

\section{NANOMATERIALS FOR ENERGY STORAGE}

Besides the high efficient conversion of the solar energy, the storage of the converted energy is also critical desired, because the night or cloudy weather can interrupt solar energy's steadiness. We should capture and store the solar energy for the usage during the interruptions of the sun light. So, energy storage is very important for the efficient consumption of energy sources. As one of the most important constituent part, the nanomaterials are closely related to the energy conversion and storage. Owing to the innovation and advancement of materials science, the energy storage nanotechnologies have also been well-developed in the decades, especially the researches on hydrogen storage and Li-ion batteries.

Efficient hydrogen storage is regarded as the key challenge in large-scale applications of hydrogen energy. Hydrogen storage materials are the core technology for the storage of hydrogen sources with efficient and safe manner. To meet the stringent requirements of application of hydrogen energy, people has devoted many efforts to develop the potential materials for hydrogen storage (Liu et al., 2010). By setting up new reaction routes, several novel systems with well thermodynamic stability were developed based the hybrid nanomaterials. The intrinsic binding states can be tuned by substituting the cation/anion in host structures, which can induced the modification of the dehydrogenation thermodynamics and kinetics (Kang et al., 2008; Xiong et al., 2008; Hügle et al., 2009; Wang et al., 2009). For example, Wang et al. found that the peak dehydrogenation temperature can be effectively decreased by introducing potassium salts in the kinetic modification study of a Li-Mg-N-H material (Wang et al., 2009). By lowering the activation energy, catalytic activation also play a versatile and 
efficient role in the enhancement of the kinetically stability of the hydrogen sorption rate at the interface of solid, gas and liquid (Bluhm et al., 2006; Denney et al., 2006; Yao et al., 2007; Gunaydin et al., 2008; Yang et al., 2008; Berseth et al., 2009). Different from the heterogeneous catalysis based hydrogen storage materials, the more efficient homogeneous catalysis is characterized by molecule-level dispersion of catalyst within the host material, which can realized by directly dissolving ammonia borane into solvents. Denney et al.'s research result shows that the dehydrocoupling temperature of ammonia borane in THF, polyether etc. organic solutions can effectively decreased (typically $45-60^{\circ} \mathrm{C}$ ) in the presence of transitionmetal complexes (Denney et al., 2006). However, the separation processes in homogeneous catalysis are more complicated than that of the heterogeneous catalysis. To overcome the kinetic barrier in the mass transport and enhance the thermodynamic, the nanoscaling can provide an effective way (Wagemans et al., 2005; Li et al., 2007; Baldé et al., 2008). Balde et al. established the relationship between size and dehydrogenation performance in the carbon nanofibers supported $\mathrm{NaAlH}_{4}$ nanoparticles. With the decrease of particle size from $1-10 \mathrm{~mm}$ to $2-10 \mathrm{~nm}$, the active energy can be successively reduced from 116 to $58 \mathrm{~kJ} \mathrm{~mol}^{-1} \mathrm{H}_{2}$ (Baldé et al., 2008). With the assistant of the nano-confinement, the dilemma between the high capacity hydrogen storage and low dehydrogenation temperature may be addressed (Gutowska et al., 2005; Ingleson et al., 2009; Kim et al., 2009). Beside the chemisorption for the hydrogen storage, the physisorption possess the superiority of facile reversible storage at ambient temperature. The morphology and surface modification is crucial in the physisorption for achieving high hydrogen capacity (McKeown et al., 2006; Dinca and Long, 2007; Yang et al., 2007; Hamaed et al., 2008; Vitillo et al., 2008; Tsao et al., 2009). It has been demonstrated that a responsible pore diameter for the efficient hydrogen storage at liquid $\mathrm{N}_{2}$ temperature and ambient pressure is $<1 \mathrm{~nm}$. For example, the carbon materials (synthesized by using zeolite as the template) with surface area as high as $3,200 \mathrm{~m}^{2} / \mathrm{g}$ and narrow pore size distribution below $1 \mathrm{~nm}$ exhibit a high hydrogen binging energy of $8.2 \mathrm{~kJ} / \mathrm{mol}$ (Yang et al., 2007). The last but not the least, the volumetric/weight hydrogen density, energy efficiency, thermodynamics, and kinetics of de-/rehydrogenation safety, cost etc. are other important factors need to overall considered for both chemisorption and physisorption in the developments of novel materials, nanostructures, and efficient synthetic techniques and strategies (Liu et al., 2010).

Li-ion batteries is one of the most important and widely used secondary batteries for the energy storage. The higher power/energy density, high speed recharge/discharge, and longer cycling life is much important for the newly emerging electronic devices, advanced communication and transportation facilities. In the past decades, many efforts were devoted to the development of the electrode materials with desirable electrochemical properties, including larger Li storage capacity, better cycling performance, and higher rate capability (Chen and Cheng, 2009). With the assistant of nanostructured materials with reduced Li-ion diffusion length and alleviated inner stress, the performance of the Li-ion batteries can be greatly improved in the rate capability and cyclability (Lee et al., 2003; Deng and Lee, 2008; Guo et al., 2008). It is very important for the development of the advanced Li-ion batteries to study the relationship between the performance and composition/nanostructure of an electrode material from the view of both theory and experiment. The compatibility of the electrode materials with electrolytes, redox sites, and surface conductivity can be greatly improved by surface engineering of the electrode materials, which further result in the improved electrochemical performance (Liu et al., 2010; Sun et al., 2016). On the other hand, rate capability and cycling performance can also be improved by growing the active nanomaterials directly on a current collector with enhanced electrical conductivity and bonding of the components (Zhang et al., 2006; Chan et al., 2008; Liu et al., 2010). Accompanied by the notable advantages, the shortcomings are also along with the subtly designed electrode nanomaterials, including large irreversible capacity, low packing density, complex synthesis processes, high cost, and so on, which further result in the limited practical applications until now. The major challenges at the material and electrode levels were large volume expansion and fracture, unstable SEI, slow electron/ion transport rate and movements of electrode atoms/molecules (Sun et al., 2016). Future works on understanding the fundamental electrode and materials chemistry taking place in these electrode systems are needed. Detailed information about the electrochemical mechanisms involved in these battery systems is still absent due to their complexity. Meanwhile, investigation of the ion and electron kinetic transport at the electrode/electrolyte interface is also important (Lin et al., 2014; Wang et al., 2014; Li et al., 2015).

\section{NANOMATERIALS FOR BIOMEDICINE}

Theranostics is a term derived from therapy and imaging, which provide an integrated platform for the personalized medicine to meet the challenges in modern health care (Chen et al., 2011; Lammers et al., 2011). The theranostics is quite related to the biocompatible nanoparticle based nanomedicine, which contain both imaging and therapeutic nanocomponents. The radio-, gene-, or chemo therapeutics may be integrated in one nanoparticles. After combine it with the intrinsic optical, magnetic, etc. physicochemical properties or appropriate biomarkers, the nanocomposites would not only allow us to diagnose disease, but also evaluate treatment efficacy by track the nanoparticles' pharmacokinetics and the drugs releasing (Prabhu and Patravale, 2012; Li et al., 2014; Muthu et al., 2014).

The nano-theranostics will face a series of biological barriers during circulation in living subjects which will influence the nanoparticle delivery efficacy: the nanoparticles firstly should cross blood vessels, then escape the entrapment of organs and removal of phagocytic cells, finally reach the specific target (Kievit and Zhang, 2011; Blanco et al., 2015). An ideal theranostic nanoparticle should possess the following characters: rapid, selective, and high efficient accumulation in target diseased tissues; feedback the detailed information (biochemical, morphological, etc.) about the interest tissues or organs; release 
the guests (drugs, chemicals, etc.) with a controllable manner for the effective therapy; easy metabolism according to a safe with less side effects after its function completed.

It has been demonstrated that the circulation and metabolism in living subjects are profoundly effected by the Size (Tang et al., 2014), shape (Shah et al., 2011; Toy et al., 2014), rigidity (Ghassemi et al., 2012), charge (Fröhlich, 2012), and surface chemistry (Mout et al., 2012) of the nanoparticles (Albanese et al., 2012; Blanco et al., 2015). The theranostic nanoparticles are the complex of the delivery carriers and cargo, targeting ligands, and bio-imaging labels, which means that the clinical translation is nontrivial for these fancy materials (Ambrogio et al., 2011; Chow and Ho, 2013). There are many factors need to considered: the prerequisite robust scale-up synthesis; the biological responses for the theranostic nanoparticles including exposure levels, systemic accumulation, excretion profiles, tissue and organ distributions of test living subjects; the potential toxicity of the nanoparticle in short and long term (Prabhu and Patravale, 2012; Muthu et al., 2014).

\section{CONCLUSIONS}

In the past decade, various nanostructures have been fabricated to address the significant material and applications challenges that exist in energy, environment, and health. Although, there are diverse specific requirements for nanomaterials in different applications, many commonality criterions for the research in the nanoscience can be built and summarized as following:

\section{REFERENCES}

Albanese, A., Tang, P. S., and Chan, W. C. (2012). The effect of nanoparticle size, shape, and surface chemistry on biological systems. Annu. Rev. Biomed. Eng. 14, 1-16. doi: 10.1146/annurev-bioeng-071811-150124

Ambrogio, M. W., Thomas, C. R., Zhao, Y. L., Zink, J. I., and Stoddart, J. F. (2011). Mechanized silica nanoparticles: a new frontier in theranostic nanomedicine. Acc. Chem. Res. 44, 903-913. doi: 10.1021/ar200018x

Baldé, C. P., Hereijgers, B. P., Bitter, J. H., and de Jong, K. P. (2008). Sodium alanate nanoparticles- linking size to hydrogen storage properties. J. Am. Chem. Soc. 130, 6761-6765. doi: 10.1021/ja710667v

Beard, M. C., Luther, J. M., and Nozik, A. J. (2014). The promise and challenge of nanostructured solar cells. Nat. Nanotechnol. 9, 951-954. doi: 10.1038/nnano.2014.292

Beard, M. C., Luther, J. M., Semonin, O. E., and Nozik, A. J. (2012). Third generation photovoltaics based on multiple exciton generation in quantum confined semiconductors. Acc. Chem. Res. 46, 1252-1260. doi: $10.1021 / \operatorname{ar} 3001958$

Beard, M. C., Midgett, A. G., Law, M., Semonin, O. E., Ellingson, R. J., and Nozik, A. J. (2009). Variations in the quantum efficiency of multiple exciton generation for a series of chemically treated PbSe nanocrystal films. Nano Lett. 9, 836-845. doi: 10.1021/nl80 $3600 \mathrm{v}$

Berseth, P. A., Harter, A. G., Zidan, R., Blomqvist, A., Araújo, C. M., Scheicher, R. H., et al. (2009). Carbon nanomaterials as catalysts for hydrogen uptake and release in NaAlH4. Nano Lett. 9, 1501-1505. doi: 10.1021/nl803498e

Blanco, E., Shen, H., and Ferrari, M. (2015). Principles of nanoparticle design for overcoming biological barriers to drug delivery. Nat. Biotechnol. 33, 941-951. doi: $10.1038 /$ nbt.3330

Bluhm, M. E., Bradley, M. G., Butterick, R., Kusari, U., and Sneddon, L. G. (2006). Amineborane-based chemical hydrogen storage: enhanced ammonia
(1) The structure-activity relationship between the nanomaterials and applications should be built firstly, which is critical for the design of the nanomaterials. A good performance in the application is our ultimate aim, so we should know what kind of materials are good for the improvement of the capability.

(2) As the core components of nanotechnology, nanomaterials provides basic building blocks for fabricating complex devices with abundant functions. To better service for building up of the structure-activity relationship, the controllable targeting synthesis is another criterion in the nanoscience research.

(3) Exploring synthetic routes to large-scale production with low cost is very important for the wide spread promotion of the new nanotechnology.

(4) Last but not least, systemically evaluation on the toxicity and environmental risks of nanomaterials is essential.

\section{AUTHOR CONTRIBUTIONS}

The author confirms being the sole contributor of this work and approved it for publication.

\section{ACKNOWLEDGMENTS}

This work was supported by the NSFC (Grant No. 21210004), China National Key Basic Research Program (Grant No. 2017YFA0207303) and Shanghai Shuguang Program (Grant No. 14SG02).

borane dehydrogenation in ionic liquids. J. Am. Chem. Soc. 128, 7748-7749. doi: 10.1021/ja062085v

Chan, C. K., Peng, H., Liu, G., McIlwrath, K., Zhang, X. F., Huggins, R. A., et al. (2008). High-performance lithium battery anodes using silicon nanowires. Nat. Nanotechnol. 3, 31-35. doi: 10.1038/nnano.2007.411

Chen, J., and Cheng, F. (2009). Combination of lightweight elements and nanostructured materials for batteries. Acc. Chem. Res. 42, 713-723. doi: 10.1021/ar800229g

Chen, X., Gambhir, S. S., and Cheon, J. (2011). Theranostic nanomedicine. Acc. Chem. Res. 44, 841-841. doi: 10.1021/ar200231d

Choi, J. J., Wenger, W. N., Hoffman, R. S., Lim, Y. F., Luria, J., Jasieniak, J., et al. (2011). Solution-processed nanocrystal quantum dot tandem solar cells. $A d v$. Mater. 23, 3144-3148. doi: 10.1002/adma.201100723

Chow, E. K., and Ho, D. (2013). Cancer nanomedicine: from drug delivery to imaging. Sci. Transl. Med. 5:216rv214. doi: 10.1126/scitranslmed.3005872

Chuang, C. H., Brown, P. R., Bulović, V., and Bawendi, M. G. (2014). Improved performance and stability in quantum dot solar cells through band alignment engineering. Nat. Mater. 13, 796. doi: 10.1038/nmat3984

Cirloganu, C. M., Padilha, L. A., Lin, Q., Makarov, N. S., Velizhanin, K. A., Luo, H., et al. (2014). Enhanced carrier multiplication in engineered quasi-type-II quantum dots. Nat. Commun. 5, 4148. doi: 10.1038/ncomms5148

Deng, D., and Lee, J. Y. (2008). Hollow core-shell mesospheres of crystalline SnO2 nanoparticle aggregates for high capacity $\mathrm{Li}+$ ion storage. Chem. Mater. 20, 1841-1846. doi: 10.1021/cm7030575

Denney, M. C., Pons, V., Hebden, T. J., Heinekey, D. M., and Goldberg, K. I. (2006). Efficient catalysis of ammonia borane dehydrogenation. J. Am. Chem. Soc. 128, 12048-12049. doi: 10.1021/ja062419g

Dinca, M., and Long, J. R. (2007). High-enthalpy hydrogen adsorption in cation-exchanged variants of the microporous metal-organic framework Mn3[(Mn4Cl)3(BTT)8(CH3OH)10]2. J. Am. Chem. Soc. 129, 11172-11176. doi: $10.1021 / \mathrm{ja} 072871 \mathrm{f}$ 
Fröhlich, E. (2012). The role of surface charge in cellular uptake and cytotoxicity of medical nanoparticles. Int. J. Nanomed. 7, 5577. doi: 10.2147/IJN.S36111

Garnett, E., and Yang, P. (2010). Light trapping in silicon nanowire solar cells. Nano Lett. 10, 1082-1087. doi: 10.1021/nl100161z

Ge, J., and Yin, Y. (2011). Responsive photonic crystals. Angew. Chem. Int. Ed. 50, 1492-1522. doi: 10.1002/anie.200907091

Ghassemi, S., Meacci, G., Liu, S., Gondarenko, A. A., Mathur, A., RocaCusachs, P., et al. (2012). Cells test substrate rigidity by local contractions on submicrometer pillars. Proc. Natl. Acad. Sci. U.S.A. 109, 5328-5333. doi: 10.1073/pnas.1119886109

Green, M. A., Emery, K., Hishikawa, Y., Warta, W., and Dunlop, E. D. (2015). Solar cell efficiency tables (Version 45). Prog. Photov. 23, 1-9. doi: 10.1002/pip.2573

Gunaydin, H., Houk, K. N., and Ozolins, V. (2008). Vacancy-mediated dehydrogenation of sodium alanate. Proc. Natl. Acad. Sci. U.S.A. 105, 3673-3677. doi: 10.1073/pnas.0709224105

Guo, Y. G., Hu, J. S., and Wan, L. J. (2008). Nanostructured materials for electrochemical energy conversion and storage devices. Adv. Mater. 20, 2878-2887. doi: 10.1002/adma.200800627

Gutowska, A., Li, L., Shin, Y., Wang, C. M., Li, X. S., Linehan, J. C., et al. (2005). Nanoscaffold mediates hydrogen release and the reactivity of ammonia borane. Angew. Chem. Int. Ed. 44, 3578-3582. doi: 10.1002/anie.200462602

Hamaed, A., Trudeau, M., and Antonelli, D. M. (2008). H2 Storage materials $(22 \mathrm{KJ} / \mathrm{mol})$ using organometallic Ti fragments as $\sigma-\mathrm{H} 2$ binding sites. J. Am. Chem. Soc. 130, 6992-6999. doi: 10.1021/ja710288g

Hügle, T., Kühnel, M. F, and Lentz, D. (2009). Hydrazine borane: a promising hydrogen storage material. J. Am. Chem. Soc. 131, 7444-7446. doi: $10.1021 /$ ja9013437

Ingleson, M. J., Barrio, J. P., Bacsa, J., Steiner, A., Darling, G. R., Jones, J. T., et al. (2009). Magnesium borohydride confined in a metal-organic framework: a preorganized system for facile arene hydroboration. Angew. Chem. Int. Ed. 48, 2012-2016. doi: 10.1002/anie.200804196

Kang, X., Fang, Z., Kong, L., Cheng, H., Yao, X., Lu, G., et al. (2008). Ammonia borane destabilized by lithium hydride: an advanced on-board hydrogen storage material. Adv. Mater. 20, 2756-2759. doi: 10.1002/adma.2007 02958

Kelzenberg, M. D., Boettcher, S. W., Petykiewicz, J. A., Turner-Evans, D. B., Putnam, M. C., Warren, E. L., et al. (2010). Enhanced absorption and carrier collection in Si wire arrays for photovoltaic applications. Nat. Mater. 9, 239-244. doi: $10.1038 /$ nmat 2727

Kievit, F. M., and Zhang, M. (2011). Cancer nanotheranostics: improving imaging and therapy by targeted delivery across biological barriers. Adv. Mater. 23, H217-H247. doi: 10.1002/adma.201102313

Kim, H., Karkamkar, A., Autrey, T., Chupas, P., and Proffen, T. (2009). Determination of structure and phase transition of light element nanocomposites in mesoporous silica: case study of NH3BH3 in MCM-41. J. Am. Chem. Soc. 131, 13749-13755. doi: 10.1021/ja904901d

Krogstrup, P., Jørgensen, H. I., Heiss, M., Demichel, O., Holm, J. V., Aagesen, M., et al. (2013). Single-nanowire solar cells beyond the Shockley-Queisser limit. Nat. Photonics 7, 306-310. doi: 10.1038/nphoton.2013.32

Lammers, T., Aime, S., Hennink, W. E., Storm, G., and Kiessling, F. (2011). Theranostic nanomedicine. Acc. Chem. Res. 44, 1029-1038. doi: $10.1021 /$ ar200019c

Lee, K. T., Jung, Y. S., and Oh, S. M. (2003). Synthesis of tin-encapsulated spherical hollow carbon for anode material in lithium secondary batteries. J. Am. Chem. Soc. 125, 5652-5653. doi: 10.1021/ja0345524

Li, W., Li, C., Ma, H., and Chen, J. (2007). Magnesium nanowires: enhanced kinetics for hydrogen absorption and desorption. J. Am. Chem. Soc. 129, 6710-6711. doi: $10.1021 / \mathrm{ja} 071323 \mathrm{z}$

Li, W., Yang, Y., Zhang, G., and Zhang, Y. W. (2015). Ultrafast and directional diffusion of lithium in phosphorene for high-performance lithium-ion battery. Nano Lett. 15, 1691-1697. doi: 10.1021/nl504336h

Li, Y., Lin, T. Y., Luo, Y., Liu, Q., Xiao, W., Guo, W., et al. (2014). A smart and versatile theranostic nanomedicine platform based on nanoporphyrin. Nat. Commun. 5, 4712. doi: 10.1038/ncomms5712

Lin, F., Markus, I. M., Nordlund, D., Weng, T. C., Asta, M. D., Xin, H. L., et al. (2014). Surface reconstruction and chemical evolution of stoichiometric layered cathode materials for lithium-ion batteries. Nat. Commun. 5, 3529. doi: $10.1038 /$ ncomms 4529
Linic, S., Christopher, P., and Ingram, D. B. (2011). Plasmonic-metal nanostructures for efficient conversion of solar to chemical energy. Nat. Mater. 10, 911. doi: 10.1038/nmat3151

Liu, C., Li, F., Ma, L. P., and Cheng, H. M. (2010). Advanced materials for energy storage. Adv. Mater. 22, E28-E62. doi: 10.1002/adma.200903328

Masuko, K., Shigematsu, M., Hashiguchi, T., Fujishima, D., Kai, M., Yoshimura, $\mathrm{N}$., et al. (2014). Achievement of more than $25 \%$ conversion efficiency with crystalline silicon heterojunction solar cell. IEEE J. Photov. 4, 1433-1435. doi: 10.1109/JPHOTOV.2014.2352151

McKeown, N. B., Gahnem, B., Msayib, K. J., Budd, P. M., Tattershall, C. E., Mahmood, K., et al. (2006). Towards polymer-based hydrogen storage materials: engineering ultramicroporous cavities within polymers of intrinsic microporosity. Angew. Chem. Int. Ed. 45, 1804-1807. doi: $10.1002 /$ anie. 200504241

Mei, A., Li, X., Liu, L., Ku, Z., Liu, T., Rong, Y., et al. (2014). A hole-conductorfree, fully printable mesoscopic perovskite solar cell with high stability. Science 345, 295-298. doi: 10.1126/science. 1254763

Mout, R., Moyano, D. F., Rana, S., and Rotello, V. M. (2012). Surface functionalization of nanoparticles for nanomedicine. Chem. Soc. Rev. 41, 2539-2544. doi: $10.1039 / \mathrm{c} 2 \mathrm{cs} 15294 \mathrm{k}$

Muthu, M. S., Leong, D. T., Mei, L., and Feng, S. S. (2014). Nanotheranostics? application and further development of nanomedicine strategies for advanced theranostics. Theranostics 4, 660. doi: 10.7150/thno.8698

Ning, Z., Voznyy, O., Pan, J., Hoogland, S., Adinolfi, V., Xu, J., et al. (2014). Air-stable n-type colloidal quantum dot solids. Nat. Mater. 13, 822. doi: $10.1038 /$ nmat 4007

Nozik, A. J., Beard, M. C., Luther, J. M., Law, M., Ellingson, R. J., and Johnson, J. C. (2010). Semiconductor quantum dots and quantum dot arrays and applications of multiple exciton generation to third-generation photovoltaic solar cells. Chem. Rev. 110, 6873-6890. doi: 10.1021/cr900289f

Oh, J., Yuan, H. C., and Branz, H. M. (2012). An 18.2\%-efficient black-silicon solar cell achieved through control of carrier recombination in nanostructures. Nat. Nanotechnol. 7, 743-748. doi: 10.1038/nnano.2012.166

Prabhu, P., and Patravale, V. (2012). The upcoming field of theranostic nanomedicine: an overview. J. Biomed. Nanotechnol. 8, 859-882. doi: 10.1166/jbn.2012.1459

Reece, S. Y., Hamel, J. A., Sung, K., Jarvi, T. D., Esswein, A. J., Pijpers, J. J., et al. (2011). Wireless solar water splitting using silicon-based semiconductors and earth-abundant catalysts. Science 334, 645-648. doi: 10.1126/science.1209816

Shah, S., Liu, Y., Hu, W., and Gao, J. (2011). Modeling particle shapedependent dynamics in nanomedicine. J. Nanosci. Nanotechnol. 11, 919-928. doi: 10.1166/jnn.2011.3536

Shockley, W., and Queisser, H. J. (1961). Detailed balance limit of efficiency of p-n junction solar cells. J. Appl. Phys. 32, 510-519. doi: 10.1063/1.1736034

Sun, Y., Liu, N., and Cui, Y. (2016). Promises and challenges of nanomaterials for lithium-based rechargeable batteries. Nat. Energy 1:16071. doi: 10.1038/nenergy.2016.71

Tang, L., Yang, X., Yin, Q., Cai, K., Wang, H., Chaudhury, I., et al. (2014). Investigating the optimal size of anticancer nanomedicine. Proc. Natl. Acad. Sci. U.S.A. 111, 15344-15349. doi: 10.1073/pnas.1411499111

Thomann, I., Pinaud, B. A., Chen, Z., Clemens, B. M., Jaramillo, T. F., and Brongersma, M. L. (2011). Plasmon enhanced solar-to-fuel energy conversion. Nano Lett. 11, 3440-3446. doi: 10.1021/nl201908s

Toy, R., Peiris, P. M., Ghaghada, K. B., and Karathanasis, E. (2014). Shaping cancer nanomedicine: the effect of particle shape on the in vivo journey of nanoparticles. Nanomedicine 9, 121-134. doi: 10.2217/nnm.13.191

Tsao, C. S., Yu, M. S., Wang, C. Y., Liao, P. Y., Chen, H. L., Jeng, U. S., et al. (2009). Nanostructure and hydrogen spillover of bridged metal-organic frameworks. J. Am. Chem. Soc. 131, 1404-1406. doi: 10.1021/ja802741b

Vitillo, J. G., Regli, L., Chavan, S., Ricchiardi, G., Spoto, G., Dietzel, P. D., et al. (2008). Role of exposed metal sites in hydrogen storage in MOFs. J. Am. Chem. Soc. 130, 8386-8396. doi: 10.1021/ja8007159

Wagemans, R. W., van Lenthe, J. H., de Jongh, P. E., Van Dillen, A. J., and de Jong, K. P. (2005). Hydrogen storage in magnesium clusters: quantum chemical study. J. Am. Chem. Soc. 127, 16675-16680. doi: 10.1021/ja054569h

Wang, J., Liu, T., Wu, G., Li, W., Liu, Y., Araújo, C. M., et al. (2009). Potassiummodified $\mathrm{Mg}(\mathrm{NH} 2) 2 / 2 \mathrm{LiH}$ system for hydrogen storage. Angew. Chem. Int. Ed. 48, 5828-5832. doi: 10.1002/anie.200805264 
Wang, X., Koleilat, G. I., Tang, J., Liu, H., Kramer, I. J., Debnath, R., et al. (2011). Tandem colloidal quantum dot solar cells employing a graded recombination layer. Nat. Photonics 5, 480-484. doi: 10.1038/nphoton.2011.123

Wang, Z., Dong, Y., Li, H., Zhao, Z., Wu, H. B., Hao, C., et al. (2014). Enhancing lithium-sulphur battery performance by strongly binding the discharge products on amino-functionalized reduced graphene oxide. Nat. Commun. 5, 5002. doi: $10.1038 /$ ncomms6002

Xiong, Z., Yong, C. K., Wu, G., Chen, P., Shaw, W., Karkamkar, A., et al. (2008). High-capacity hydrogen storage in lithium and sodium amidoboranes. Nat. Mater. 7, 138-141. doi: 10.1038/nmat2081

Yang, J., Sudik, A., Siegel, D. J., Halliday, D., Drews, A., Carter, R. O., et al. (2008). A self-catalyzing hydrogen-storage material. Angew. Chem. Int. Ed. 47, 882-887. doi: $10.1002 /$ anie. 200703756

Yang, Z., Xia, Y., and Mokaya, R. (2007). Enhanced hydrogen storage capacity of high surface area zeolite-like carbon materials. J. Am. Chem. Soc. 129, 1673-1679. doi: $10.1021 / \mathrm{ja} 067149 \mathrm{~g}$

Yao, X., Wu, C., Du, A., Zou, J., Zhu, Z., Wang, P., et al. (2007). Metallic and carbon nanotube-catalyzed coupling of hydrogenation in magnesium. J. Am. Chem. Soc. 129, 15650-15654. doi: 10.1021/ja0751431
Zhang, H.-L., Zhang, Y., Zhang, X.-G., Li, F., Liu, C., Tan, J., et al. (2006). Urchinlike nano/micro hybrid anode materials for lithium ion battery. Carbon 44, 2778-2784. doi: 10.1016/j.carbon.2006.03.029

Zhang, J., Gao, J., Miller, E. M., Luther, J. M., and Beard, M. C. (2013). Diffusion-controlled synthesis of $\mathrm{PbS}$ and $\mathrm{PbSe}$ quantum dots with in situ halide passivation for quantum dot solar cells. ACS Nano 8, 614-622. doi: $10.1021 / \mathrm{nn} 405236 \mathrm{k}$

Conflict of Interest Statement: The author declares that the research was conducted in the absence of any commercial or financial relationships that could be construed as a potential conflict of interest.

Copyright (c) 2017 Zhang. This is an open-access article distributed under the terms of the Creative Commons Attribution License (CC BY). The use, distribution or reproduction in other forums is permitted, provided the original author(s) or licensor are credited and that the original publication in this journal is cited, in accordance with accepted academic practice. No use, distribution or reproduction is permitted which does not comply with these terms. 